\title{
Crystallinity of Electrospun and Centrifugal Spun Polycaprolactone Fibers: A Comparative Study
}

\author{
Eva Kuzelova Kostakova, ${ }^{1}$ Laszlo Meszaros, ${ }^{2,3}$ Gabriela Maskova, ${ }^{1}$ Lenka Blazkova, \\ Tamas Turcsan, ${ }^{2}$ and David Lukas ${ }^{1}$ \\ ${ }^{1}$ Department of Nonwovens and Nanofibrous Materials, Faculty of Textile Engineering, Technical University of Liberec, \\ Studentska 2, 46117 Liberec 1, Czech Republic \\ ${ }^{2}$ Department of Polymer Engineering, Faculty of Mechanical Engineering, Budapest University of Technology and Economics, \\ Muegyetem Rkp. 3, T. Bldg. III, Budapest 1111, Hungary \\ ${ }^{3}$ MTA-BME Research Group for Composite Science and Technology, Muegyetem Rkp. 3, Budapest 1111, Hungary
}

Correspondence should be addressed to Eva Kuzelova Kostakova; eva.kostakova@tul.cz

Received 27 September 2016; Accepted 1 February 2017; Published 5 March 2017

Academic Editor: Russell E. Gorga

Copyright ( 92017 Eva Kuzelova Kostakova et al. This is an open access article distributed under the Creative Commons Attribution License, which permits unrestricted use, distribution, and reproduction in any medium, provided the original work is properly cited.

Crystalline properties of semicrystalline polymers are very important parameters that can influence the application area. The internal structure, like the mentioned crystalline properties, of polymers can be influenced by the production technology itself and by changing technology parameters. The present work is devoted to testing of electrospun and centrifugal spun fibrous and nanofibrous materials and compare them to foils and granules made from the same raw polymer. The test setup reveals the structural differences caused by the production technology. Effects of average molecular weight are also exhibited. The applied biodegradable and biocompatible polymer is polycaprolactone (PCL) as it is a widespread material for medical purposes. The crystallinity of PCL has significant effect on rate of degradation that is an important parameter for a biodegradable material and determines the applicability. The results of differential scanning calorimetry (DSC) showed that, at the degree of crystallinity, there is a minor difference between the electrospun and centrifugal spun fibrous materials. However, the significant influence of polymer molecular weight was exhibited. The morphology of the fibrous materials, represented by fiber diameter, also did not demonstrate any connection to final measured crystallinity degree of the tested materials.

\section{Introduction}

Electrospinning and centrifugal spinning are widespread technologies for production of fibrous and nanofibrous materials nowadays. The final products are partiality used for medical applications mainly as drug delivery systems, wound dressings, and scaffolds for tissue engineering. The applied polymeric materials are usually biodegradable, which are typically semicrystalline ones, thus consisting of amorphous and crystalline phases. The polymer in the amorphous state has an unordered structure contrary to the crystalline material that exhibits orderliness. The arranged areas are called crystallites and their sizes are generally of hundreds of nanometers [1]. The crystalline phase has a higher density and stiffness compared to the amorphous phase. The crystallinity of the same raw polymer material differs with the use of different treatment or production technologies [1].
In some studies, it is indicated that electrospun fibers from polymer solutions have a lower crystallinity than fibers produced from melts or foils from dilute solutions. The cause of lower crystallinity is usually explained by the relatively quick deposition of fibers (the jet velocity during needle electrospinning for polyethyleneoxide/water solutions was measured from 0.5 to $5 \mathrm{~m} / \mathrm{s}$ ) onto the collector during electrospinning, which does not allow enough time to evolve high crystalline portion in the final fiber. Besides there is an opportunity to influence slightly the final crystallinity of the fibers at a given technology by varying the processing conditions [2-4].

The degree of crystallinity (crystalline ratio) is very important for a polymer material degradation point of view and it is known that the amorphous parts degrade preferably. At the beginning of degradation, crystallites appear on 
the surface of polymeric material, which may explain the mentioned preferential degradation of the amorphous phase of the polymer. Because of the more stable structure of the crystallites, there is a direct proportionality between the degradation rate and the initial crystallinity [5]. The degree of crystallinity of the fibers determines the degradation time that is one important property from an implanted material point of view. On the other hand, higher crystallinity means higher strength properties that is also basically important. The formed morphology of a semicrystalline polymer material depends on the crystallization conditions. In case of electrospinning and centrifugal spinning, these conditions include the polymer solution concentration, polymer molecular weight, and presence and type of nucleating agents. The crystallization conditions are of course connected with production technology parameters (process and material conditions).

Polycaprolactone (PCL) is a hydrophobic, semicrystalline polymer having a glass transition temperature $\left(T_{g}\right)$ of $-60^{\circ} \mathrm{C}$ and crystalline melting point ranging between 59 and $64^{\circ} \mathrm{C}$. Its crystallinity tends to decrease with increasing molecular weight, that is typical for semicrystalline polymers. PCL is soluble in chloroform, dichloromethane, carbon tetrachloride, benzene, toluene, cyclohexanone, and so forth at room temperature. This polymer has a low solubility in acetone, butanone, ethyl acetate, dimethylformamide, diethyl ether, and so on and is insoluble in alcohol, petroleum ether, diethyl ether, and so forth [6]. The PCL homopolymer has a total degradation of 2-4 years depending on the starting molecular weight of the applied material and structural properties as porosity, thickness, total specific surface, and so on. The rate of hydrolysis can be altered by copolymerization with other lactones or glycolides/lactides. The degradation accelerates in enzyme rich or acidic environment. Nowadays, typical medical usage of PCL materials is drug delivery systems and scaffolds for tissue engineering of bones, cartilages, tendon and ligaments, blood vessels, skin, and nerves and as medical devices mainly as sutures and wound dressing $[6,7]$.

If electrospinning solution technology is taken into consideration, the relevant processing parameters are applied electrical voltage, distance between spinning electrode and collector, ambient temperature and humidity, type of polymer, its molecular weight, type of solvent/solvents, and polymer solution concentration However, the degree of influence of these parameters on the crystallinity of the resulting polymer fibers is not straightforward. Ero-Phillips et al. [8] investigated the effect of the applied electric voltage at electrospinning on the crystallinity of the produced PLA nanofibers. They found that the effect of the applied voltage has strong effect on the crystallinity, which depends also significantly on the solution concentration, and the collector distances also have some effect. In contrast, Kołbuk et al. [9] exhibited just slight influence of applied electric voltage (polycaprolactone/chloroform/methanol; polycaprolactone/chloroform/dimethylformamide) to the resulting crystallinity of the electrospun fibers.

Distance between spinning electrode and collector has usually only moderate influence to the final crystallinity of an electrospun fibrous material. However, it is important that the fiber had time to elongate, organize macromolecules, and thus influence the morphology and diameter of the resulting electrospun fibers. Overall, fibers with longer trajectories have usually higher molecular orientation and their crystallinity is also higher $[8,10]$. The temperature and humidity of the ambient environment in which the process of spinning proceeds have an influence on spinning of polymer solutions and the rate of evaporation of the solvent [11]. The more volatile the solvent is, the faster the evaporation occurs in the formation of fibers and therefore the crystallinity of the formed fibers is lower. It is necessary to avoid too fast drying, or on the contrary too slow $[8,12]$.

The parameters of centrifugal spinning, which influenced the crystallinity of final fibers, are mainly peripheral velocity of spinneret, distance between spinneret and collector, solution concentration, and type of polymer and its molecular weight. Lu et al. [13] investigated the effects of the centrifugal spinning parameters on the crystallinity of polyacrylonitrile fibers using polyacrylonitrile/dimethylformamide solution as raw material. They found that the increasing peripheral velocity increased the degree of final fibers crystallinity and similar effect was exhibited by increasing distance between spinneret and collector. In contrast, increasing polymer solution concentration caused decrease in final fibrous material degree of crystallinity.

The presenting article is devoted to measure the degree of crystallinity of two fibrous materials prepared from different solutions and melts and by different technologies, investigating the effects of these parameters. The fibrous materials are compared with basic granules and foils prepared from used solutions. The article shows how the spinning technology and solution parameters influenced the final material degree of crystallinity.

\section{Materials and Methods}

The investigated material was poly- $\varepsilon$-caprolactone (PCL) in pellet form, having an average molecular weight of $M_{n}$ $=45,000 \mathrm{~g} / \mathrm{mol}$ (PCL45) and $M_{n}=80,000 \mathrm{~g} / \mathrm{mol}$ (PCL80), density $1.145 \mathrm{gcm}^{-3}$, melting point $60^{\circ} \mathrm{C}$ from Sigma Aldrich Inc. and dissolved in different solvents and solvent mixtures (chloroform, ethanol, methanol, ethyl acetate, dimethylsulfoxid (DMSO), dimethylacetamide (DMAc)). The solutions were mixed for 24 hours by a magnetic stirrer. The final solutions were as follows: (A) 16 wt\% PCL45 dissolved in chloroform/ethanol in ratio $9: 1$ by weight; (B) $12 \mathrm{wt} \%$ PCL80 dissolved in chloroform/ethanol in ratio $9: 1$ by weight; (C) 16 wt $\%$ PCL 45 dissolved in chloroform/methanol in ratio $9: 1$ by weight; (D) $7 \mathrm{wt} \%$ PCL80 dissolved in chloroform/methanol in ratio $3: 1$ by weight; (E) $24 \mathrm{wt} \%$ PCL45 dissolved in ethyl acetate/DMSO in ratio $8: 2$ by weight; (F) $24 \mathrm{wt} \%$ PCL45 dissolved in ethyl acetate/DMAc in ratio $8: 2$ by weight. These solutions were chosen according to experience from previous experiments and literature $[9,14,15]$ for their willingness to be relatively well spun by electrospinning or centrifugal spinning. Unfortunately, it was impossible to find only one concentration for spinning of all materials to compare them. 


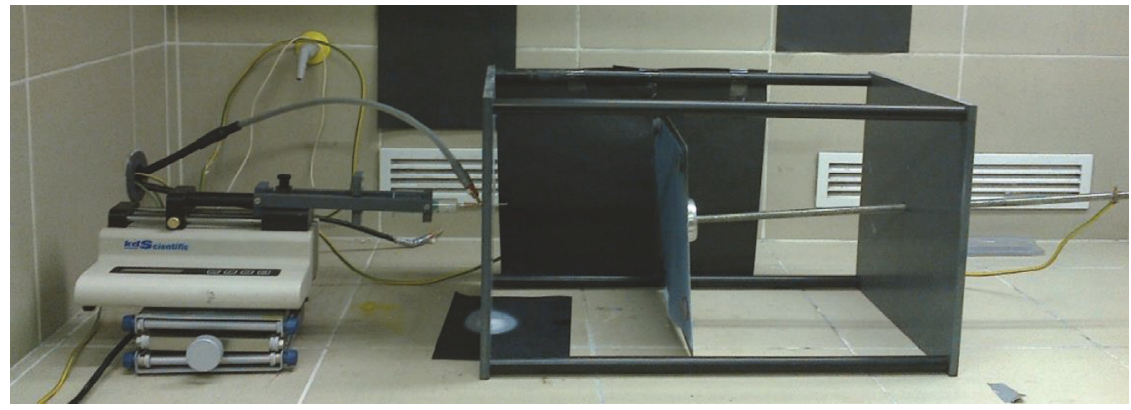

(a)
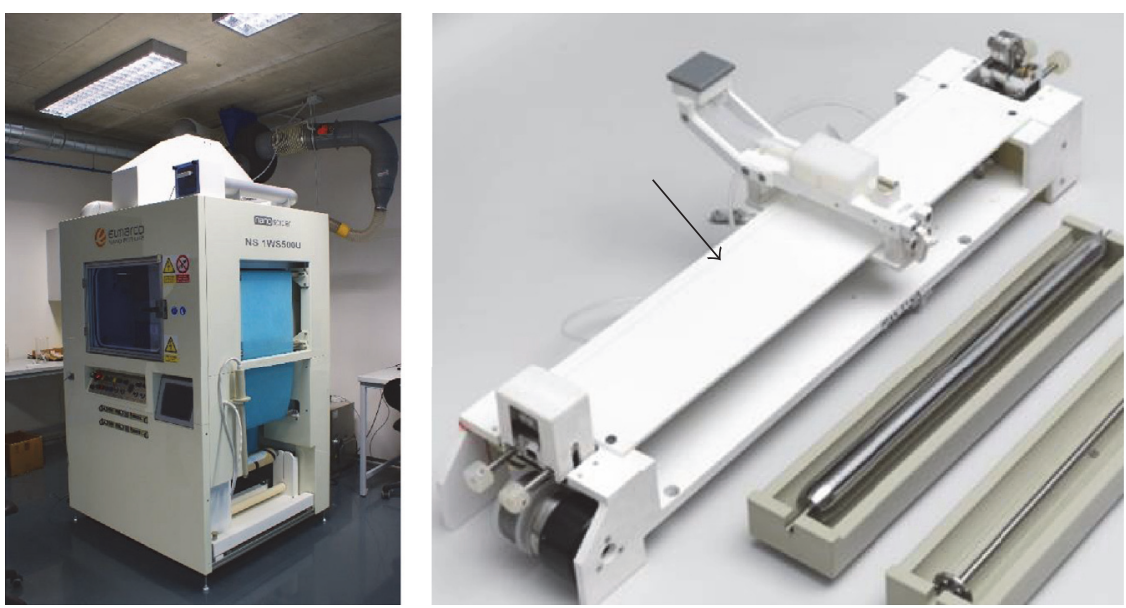

(b)

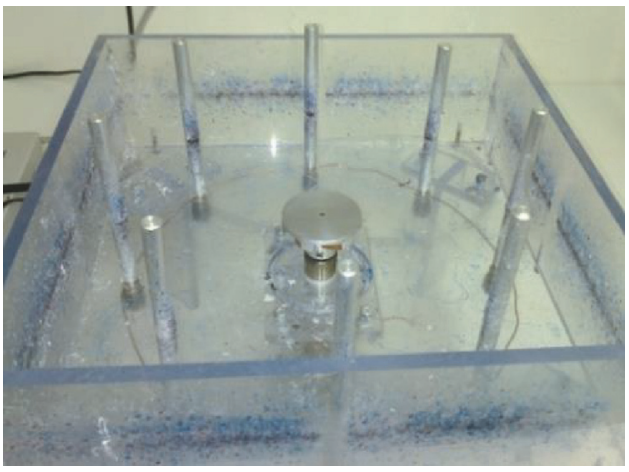

(c)

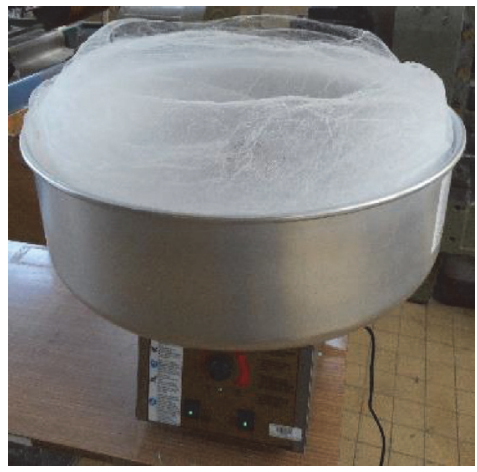

(d)

FIGURE 1: Spinning devices: (a) needle electrospinning with metal needle connected to the dosing equipment and high voltage source and grounded metal plat collector; (b) Nanospider ${ }^{\mathrm{TM}}$ for needleless electrospinning technology and string spinning electrode (arrowed); (c) centrifugal spinning laboratory device with rotating disk and eight collecting cylinders arranged in a circle; (d) machine for cotton candy production The Breeze ${ }^{\circledR} 3030 \mathrm{EX}$ used for melt centrifugal spinning.

Three different devices were used for spinning of the above-mentioned solutions: needle electrospinning, needleless electrospinning, and centrifugal spinning. The needle electrospinning apparatus (see Figure 1(a)) used for electrospinning experiments consists of a syringe equipped with a metal hypodermic needle of internal diameter of $0.5 \mathrm{~mm}$ and length of $40 \mathrm{~mm}$. The needle was connected to the positive pole of a high voltage source (Spellman SL 150) and placed in the feeding device (KDScientific KDS-100). A grounded metallic place was used as a collector. The process parameters were positive voltage of $20 \mathrm{kV}$ at the needle; the distance between needle end and the collector surface adjusted to $250 \mathrm{~mm}$; feeding velocity $2 \mathrm{mlh}^{-1}$; laboratory temperature $24 \pm 2^{\circ} \mathrm{C}$; and ambient humidity $42 \pm 5 \% \mathrm{RH}$. These samples were signed according the solution compositions A-F.

As a needleless electrospinning device (see Figure 1(b)) was used machine Nanospider NS 1WS500U (Elmarco, CZ) with string spinning electrode of $200 \mu \mathrm{m}$ diameter. The string is attached to a dosing equipment, to which was always metered at least $20 \mathrm{~mL}$ of prepared polymer solution. The 
dosing equipment was connected by a pneumatic mechanism. Contact and correct dosing of the solution onto string spinning electrode was ensured by using a die having a diameter of $700 \mu \mathrm{m}$. Other process parameters were positive voltage of $26 \pm 4 \mathrm{kV}$ at the string spinning electrode; the distance between spinning electrode and the collector surface being $190 \mathrm{~mm}$; laboratory temperature $23 \pm 1^{\circ} \mathrm{C}$; and ambient humidity $34 \pm 3 \% \mathrm{RH}$. These samples are signed according solution compositions As-Fs.

The centrifugal spinning was done with a laboratory device (see Figure 1(c)) consisting of rotating metal disk connecting with an electric engine. The central rotating disk of $50 \mathrm{~mm}$ in diameter was surrounded by eight collecting cylinders arranged in a circle in $260 \mathrm{~mm}$ in diameter. The used peripheral speed was $27 \mathrm{~m} / \mathrm{s}$. The polymer solution was dripped onto the rotating disc from $100 \mathrm{~mm}$ distance and the fibers were finally taken from collecting cylinders. Samples produced by centrifugal spinning were marked according the solution compositions Ac-Dc. The solutions E and F were not able to sufficiently spin by the centrifugal spinning technology. Also the melt centrifugal spinning technology was used for comparison with samples produced from solutions. The machine for cotton candy mass production The Breeze ${ }^{\circledR}$ 3030EX (Gold Medal Funfood Equipment and Supplies, USA) was applied for spinning of PCL45 and PCL80. The fibrous samples produced by melt centrifugal spinning were marked as X45 and X80 according the used polymer molecular weight.

All polymer solutions were also transformed into foils. The solution $(1 \mathrm{~mL})$ was poured onto an aluminum foil and left to dry at laboratory conditions (43\% RH; $\left.23^{\circ} \mathrm{C}\right)$. The dry foils were peeled away and tested as samples Af-Ff supplementary to the fibrous materials.

The crystallinity was analyzed by DSC analysis (TA Instruments, type Q2000). The DSC measurements parameters were as follows: weight of each sample was $10 \pm$ $0.5 \mathrm{mg}$; heating and cooling rate was $10^{\circ} \mathrm{C} / \mathrm{min}$; start temperature was $20^{\circ} \mathrm{C}$ and maximal temperature was $75^{\circ} \mathrm{C}$; the heating/cooling/heating regime was chosen. Five different samples from each material were measured. The values for the degree of crystallinity were calculated using the following [9]:

$$
\chi_{c}=\frac{\int_{0}^{\infty}(d H / d t) d t}{\Delta H^{0}}
$$

where $\chi_{c}$ is the degree of crystallinity, $H$ is enthalpy relative to the weight, $t$ is time, then $d H / d t$ is the rate of melting per unit mass, and $\Delta H^{0}$ is enthalpy of $100 \%$ crystalline PCL material, respectively, specific heat of fusion of $100 \%$ crystalline PCL taken as $139,5 \mathrm{~J} \cdot \mathrm{g}^{-1}[16,17]$. The DSC measurements were carried out under a nitrogen atmosphere. The crystallinity of fibers was calculated from peak representing the first heating of the sample.

Samples structures were analyzed using scanning electron microscopy (SEM; Tescan Vega S3 EasyProbe and 638LVa JEOL) and image analyzer software (Lucia G 4.82, LIM). Each specimen was coated with gold in a sputtering device Quorum Q150R ES. The average fiber diameter of each sample was determined using average value for one hundred measurements.

\section{Results and Discussion}

3.1. Fiber Morphology. Scanning electron microscopic images confirmed that solution composition and used technology changed the final fibrous structures. The main differences are in average fiber diameter and amount of defects (mainly in a form of spherical drops, beads, and thick fibers). Figure 2 illustrates chosen SEM images of needle and needleless electrospun fibers. The diameter of fibers reflects all of the parameters acting on the fiber during electrospinning (solution concentration, polymer molecular weight, electrospinning technology, etc.).

It can be seen in Figure 2 that the molecular weight has major effect on the awaking fiber structure for the needle electrospinning technology mainly. In case of samples A and $\mathrm{B}$, the solvent system is the same, and even if the PCL80 was in lower concentration (usually without the molecular weight change, it causes lower fiber diameter), the fiber diameter increased. This effect can be explained by that longer molecules have more intermolecular connections and entanglements that decrease the molecular mobility and the molecules relative movement to each other is hampered that results in larger diameter. Contrary effect was observed for chloroform/methanol solvent (Figures 2(C) and 2(D)), but significantly lower concentrations and different ratios of solvents were used for sample D. The presence of methanol instead of ethanol resulted in slightly higher average diameter (samples $\mathrm{A}$ and $\mathrm{C}$ ), what can be explained by different interactions between macromolecules inside these spinning solutions. In case of using needleless electrospinning method for the PCL80, the spinning results in lower average diameter of fibers for samples Bs and Ds compared to spun samples from PCL45 (As and Cs). The interesting phenomenon is done by bimodal fibrous structure of materials made from PCL45 as it is visible on Figures 2(As) and 2(Cs). These samples contain very thin fibers but also fibers with higher diameter. Thus, finally, the total average fiber diameter for samples from PCL45 seems higher than the average fiber diameter for PCL80. For these solvents, the technology has major effect: the average diameter is less for needleless electrospun mats than the needle spun ones. This phenomenon can be explained by the higher applied voltage and smaller collector distance for needleless electrospinning. The same production conditions for needle and needleless electrospinning could not be set because of termination one or, respectively, second electrospinning type. Overall, it causes higher repulsive forces in the spinning volume for this setup of needleless electrospinning that is favorable for forming thin fibers. Samples A (As), D (Ds), and F (Fs) do not show any significant changes in fiber diameter influenced by electrospinning technology change. Their confidence intervals overlap each other. Sample Es contained thinner fibers compared to sample E but also contained many defects; in this case, the needleless electrospinning was hardly feasible.

The final fibrous structures produced by solution and melt centrifugal spinning are introduced by chosen SEM 

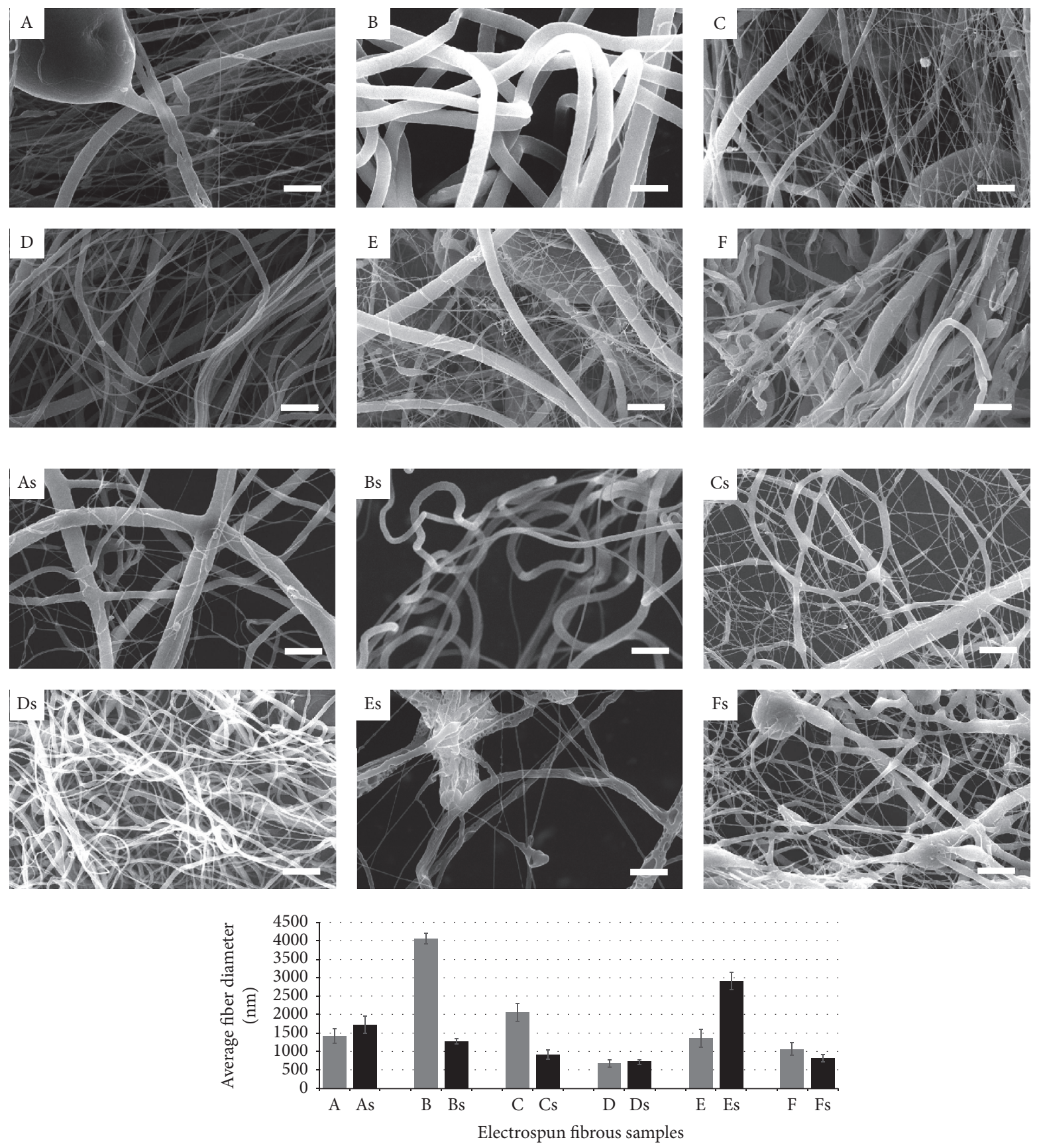

FIGURE 2: Scanning electron microscope images of chosen fibrous materials produced by needle (A-F) and needleless (As-Fs) electrospinning technology and graph representing average fiber diameter with error bars representing $95 \%$ confidence intervals. The scale bar is $10 \mu \mathrm{m}$ at all images.

images in Figure 3. Also these images confirm that solution composition, polymer molecular weight, and type of technology strongly influenced the final fibrous structures. The melt centrifugal spun materials had the highest average fiber diameter and the results correspond with the prediction that the higher molecular weight causes the higher fiber diameter. If the electrospinning methods to the nonelectrospinning ones are compared, it is clear that thinner fibers are made by electrospinning, but other methods contain less defects mainly in pearl form.
3.2. Fibrous Materials Crystallinity. The DSC analysis was used for finding the degree of crystallinity of all tested samples and crystalline melting temperature of them. Figure 4 illustrates a typical DSC scan registered during all procedure heating/cooling/heating constrained tested material at $10^{\circ} \mathrm{C} /$ minute. The DSC measurements show (see Table 1 and Figure 5) that the melting temperature of all materials did not significantly change with solution composition, molecular weight of applied polymer and technology. For the fibers, the $95 \%$ confidence interval represents the structure 

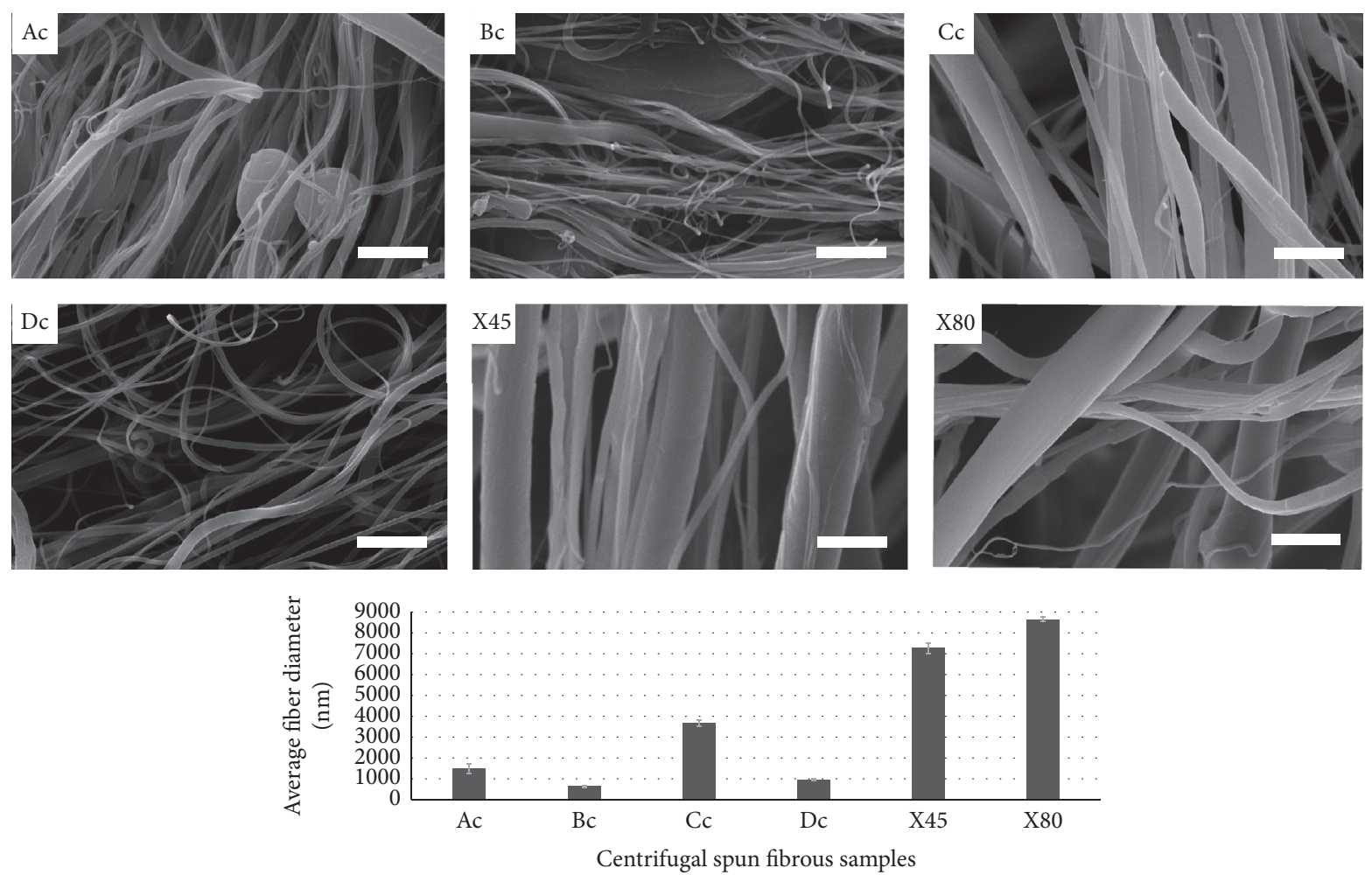

FIGURE 3: Scanning electron microscope images of chosen fibrous materials produced by centrifugal spinning from solutions (Ac-Dc) and melts (X45; X80) and graph representing average fiber diameter with error bars representing $95 \%$ confidence intervals. The scale bar is $10 \mu \mathrm{m}$ at all images.

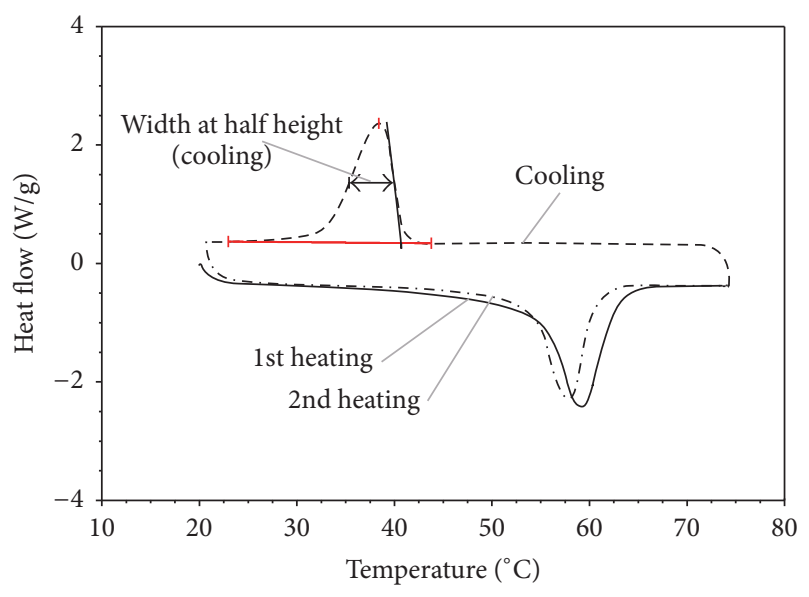

FIGURE 4: An example of DSC scan for one sample E (solution 24 wt\% PCL45 needle electrospun from ethyl acetate/DMSO $(8: 2))$ showing parts of the curve representing 1st heating, cooling, 2nd heating, and width at half height at cooling part of curve.

inhomogeneities of mats formed by electrospinning and centrifugal spinning. According to data in Table 1 and Figure 6, it is obvious that degree of crystallinity does not strongly depend on solvent composition in case of samples spun from PCL45, which is in contradiction with the results in the publication [9]. The authors used the very small quantities of material around $1 \mathrm{mg}$ for DSC testing, which could cause distortion of the results. On the contrary, the samples made from PCL80 show in all technologies (needle electrospinning, needleless electrospinning, centrifugal spinning, and foils) significantly higher degree of crystallinity for samples made from ethanol solutions than from methanol solutions. If the comparison between the crystallinity degree of origin granules and spun materials is followed, there can be found that, for the first heating, one can conclude that, in case of PCL 45 at all spinning technologies, the crystallinity decreased and, in case of the PCL 80 at the electrospinning technologies, the degree of crystallinity decreased. The data presenting in Table 1 and Figure 6 show moderate dependence of used technology to degree of crystallinity (measured for the 1st heating and representing the influence of the technology or treatment of polymer material). The strong dependence of used molecular weight of PCL to degree of spun fiber crystallinity was confirmed. The degree of crystallinity for samples made from PCL80 was in all cases (electrospun, needleless electrospun, centrifugal spun samples from polymer solutions, foils, and origin granules) except melt centrifugal spun samples (X45 and X80) that were significantly lower than for samples made from PCL45.

The sharpness of the phase transition is often expressed as the temperature width at half height [18]. Decreasing with at half height means that more perfect crystals are present, or the crystallite size distribution is reduced [19]. Usually if more perfect crystals are present, the crystalline melting 
TABLE 1: Overview of data obtained from DSC curves analysis for all tested fibrous and nonfibrous materials: $T_{m}\left({ }^{\circ} \mathrm{C}\right)$ melting temperature; $X_{c}$ (\%) degree of crystallinity calculated from the 1 st heating, cooling, and 2 nd heating and width at half height $\left({ }^{\circ} \mathrm{C}\right)$. Data represent average value and $95 \%$ confidence interval. The gray-tinged cells symbolize materials produced from raw PCL of molecular weight $M_{n}=80,000 \mathrm{~g} / \mathrm{mol}$.

\begin{tabular}{|c|c|c|c|c|c|c|c|}
\hline $\begin{array}{l}\text { PCL } \\
\text { solution }\end{array}$ & Technology & $\begin{array}{c}M_{n} \\
(\mathrm{~g} / \mathrm{mol})\end{array}$ & $\begin{array}{c}T_{m}\left({ }^{\circ} \mathrm{C}\right) \\
\text { 1st heating }\end{array}$ & $\begin{array}{c}X_{c}(\%) \\
\text { 1st heating }\end{array}$ & $\begin{array}{l}X_{c}(\%) \\
\text { cooling }\end{array}$ & $\begin{array}{c}X_{c}(\%) \\
\text { 2nd heating }\end{array}$ & $\begin{array}{c}\text { Width at half } \\
\text { height } \\
\left({ }^{\circ} \mathrm{C}\right)\end{array}$ \\
\hline A & \multirow{6}{*}{$\begin{array}{c}\text { Needle } \\
\text { electrospinning }\end{array}$} & 45,000 & $59.9 \pm 0.2$ & $63.8 \pm 1.2$ & $47.4 \pm 0.5$ & $49.3 \pm 1.1$ & $6.0 \pm 0.2$ \\
\hline B & & 80,000 & $58.3 \pm 0.2$ & $52.2 \pm 1.0$ & $36.9 \pm 0.3$ & $40.3 \pm 1.1$ & $4.8 \pm 0.6$ \\
\hline $\mathrm{C}$ & & 45,000 & $60.3 \pm 0.5$ & $62.9 \pm 2.7$ & $46.3 \pm 0.3$ & $47.5 \pm 1.6$ & $6.0 \pm 0.3$ \\
\hline $\mathrm{D}$ & & 80,000 & $59.3 \pm 0.2$ & $49.6 \pm 2.1$ & $36.8 \pm 1.7$ & $40.1 \pm 1.0$ & $7.8 \pm 0.2$ \\
\hline E & & 45,000 & $59.6 \pm 0.5$ & $63.4 \pm 1.7$ & $47.2 \pm 0.4$ & $47.7 \pm 1.6$ & $4.4 \pm 0.2$ \\
\hline $\mathrm{F}$ & & 45,000 & $60.3 \pm 0.2$ & $64.9 \pm 0.6$ & $46.4 \pm 0.7$ & $48.0 \pm 1.2$ & $4.4 \pm 1.0$ \\
\hline As & \multirow{6}{*}{$\begin{array}{l}\text { Needleless } \\
\text { electrospinning } \\
\text { (Nanospider) }\end{array}$} & 45,000 & $60.0 \pm 0.2$ & $64.8 \pm 0.9$ & $46.8 \pm 0.6$ & $49.9 \pm 1.2$ & $5.8 \pm 0.2$ \\
\hline Bs & & 80,000 & $59.5 \pm 0.3$ & $52.8 \pm 0.8$ & $35.6 \pm 0.6$ & $38.7 \pm 1.4$ & $11.6 \pm 0.5$ \\
\hline Cs & & 45,000 & $59.2 \pm 0.3$ & $62.6 \pm 0.9$ & $46.7 \pm 0.6$ & $49.7 \pm 1.3$ & $4.9 \pm 0.5$ \\
\hline Ds & & 80,000 & $59.6 \pm 0.1$ & $51.5 \pm 1.6$ & $36.6 \pm 0.6$ & $39.9 \pm 0.7$ & $7.9 \pm 0.2$ \\
\hline Es & & 45,000 & $59.0 \pm 0.1$ & $65.2 \pm 0.7$ & $44.6 \pm 1.1$ & $47.6 \pm 1.5$ & $4.7 \pm 0.3$ \\
\hline Fs & & 45,000 & $59.7 \pm 0.2$ & $64.4 \pm 1.2$ & $46.2 \pm 0.6$ & $49.7 \pm 1.2$ & $4.0 \pm 0.4$ \\
\hline Ac & \multirow{4}{*}{$\begin{array}{l}\text { Solution centrifugal } \\
\text { spinning }\end{array}$} & 45,000 & $60.3 \pm 0.3$ & $62.6 \pm 0.4$ & $50.1 \pm 0.2$ & $48.6 \pm 0.5$ & $7.0 \pm 0.3$ \\
\hline $\mathrm{Bc}$ & & 80,000 & $60.3 \pm 0.4$ & $59.3 \pm 1.3$ & $48.0 \pm 1.0$ & $46.8 \pm 0.8$ & $7.3 \pm 0.4$ \\
\hline $\mathrm{Cc}$ & & 45,000 & $60.8 \pm 0.5$ & $60.9 \pm 1.3$ & $48.3 \pm 0.8$ & $48.4 \pm 0.7$ & $6.8 \pm 0.4$ \\
\hline Dc & & 80,000 & $60.2 \pm 0.4$ & $50.0 \pm 1.0$ & $39.2 \pm 0.6$ & $38.5 \pm 1.3$ & $11.3 \pm 0.8$ \\
\hline $\mathrm{X} 45$ & \multirow{2}{*}{$\begin{array}{l}\text { Melt centrifugal } \\
\text { spinning }\end{array}$} & 45,000 & $60.7 \pm 0.5$ & $59.1 \pm 1.3$ & $48.1 \pm 0.8$ & $45.2 \pm 0.6$ & $6.4 \pm 0.6$ \\
\hline X80 & & 80,000 & $60.8 \pm 0.1$ & $58.8 \pm 0.6$ & $47.5 \pm 0.3$ & $47.6 \pm 1.1$ & $6.5 \pm 0.3$ \\
\hline PCL 45 & \multirow{2}{*}{ Granules } & 45,000 & $60.1 \pm 1.4$ & $66.4 \pm 1.9$ & $47.3 \pm 1.8$ & $47.4 \pm 2.4$ & $7.7 \pm 0.2$ \\
\hline PCL 80 & & 80,000 & $58.7 \pm 0.2$ & $56.6 \pm 0.9$ & $38.2 \pm 0.3$ & $41.7 \pm 1.6$ & $7.0 \pm 0.2$ \\
\hline Af & \multirow{6}{*}{ Foils } & 45,000 & $61.5 \pm 0.5$ & $67.2 \pm 0.8$ & $48.1 \pm 0.9$ & $49.7 \pm 1.8$ & $10.0 \pm 1.1$ \\
\hline $\mathrm{Bf}$ & & 80,000 & $60.9 \pm 0.5$ & $59.2 \pm 0.7$ & $49.5 \pm 0.4$ & $41.0 \pm 1.3$ & $8.6 \pm 0.7$ \\
\hline Cf & & 45,000 & $61.4 \pm 0.4$ & $67.7 \pm 0.4$ & $40.2 \pm 0.8$ & $48.6 \pm 0.9$ & $6.6 \pm 0.5$ \\
\hline Df & & 80,000 & $60.4 \pm 0.5$ & $57.9 \pm 0.4$ & $48.4 \pm 0.4$ & $41.3 \pm 0.8$ & $6.5 \pm 0.4$ \\
\hline Ef & & 45,000 & $64.4 \pm 0.5$ & $61.0 \pm 1.4$ & $40.0 \pm 0.3$ & $47.2 \pm 0.5$ & $6.9 \pm 0.4$ \\
\hline $\mathrm{Ff}$ & & 45,000 & $61.2 \pm 0.7$ & $69.9 \pm 2.6$ & $47.7 \pm 0.9$ & $49.2 \pm 1.6$ & $4.6 \pm 0.9$ \\
\hline
\end{tabular}

temperatures increase. In the investigated materials, there were no significant changes observed for the melting points ergo and the decreasing half height with represents more uniform crystallite size distribution that is an important parameter from the biodegradation point of view. Except for some instances (D types and Bs), one can conclude that the more homogenous crystallite size distributions could be achieved by the electrospinning technologies compared to other ones.

The melting temperatures introduced in Table 1 and Figure 5 were not influenced by the fibrous material structure. There is no connection between melting temperature and fiber diameter. It is also significant that the all the samples had the higher crystallinity at the first heating compared to the second one.

\section{Conclusions}

The measured data in the presenting study show that the process parameters (spinning technology changes) and the material parameters (solvent systems, polymer molecular weight, concentration, etc.) have a significant impact on the final morphology of the produced fibrous materials. However, the influence on the crystallinity to the spinning technology for the fiber samples was minimal. The crystallinity differed in a few percentage units only. Nevertheless, the influence of the changes in the concentration, the used solvent system, and molecular weight of the origin polymer material to the final fiber crystallinity changes have been shown. At the same time, there was no correlation found between the crystallinity of the fibers and their diameter. Investigation of internal structures of fibrous materials is very important given the prediction of their behavior in end-use applications. Detection of crystallinity is closely related to material biodegradation and therefore it is necessary to pay more attention to these studies.

\section{Competing Interests}

The authors declare that they have no competing interests. 


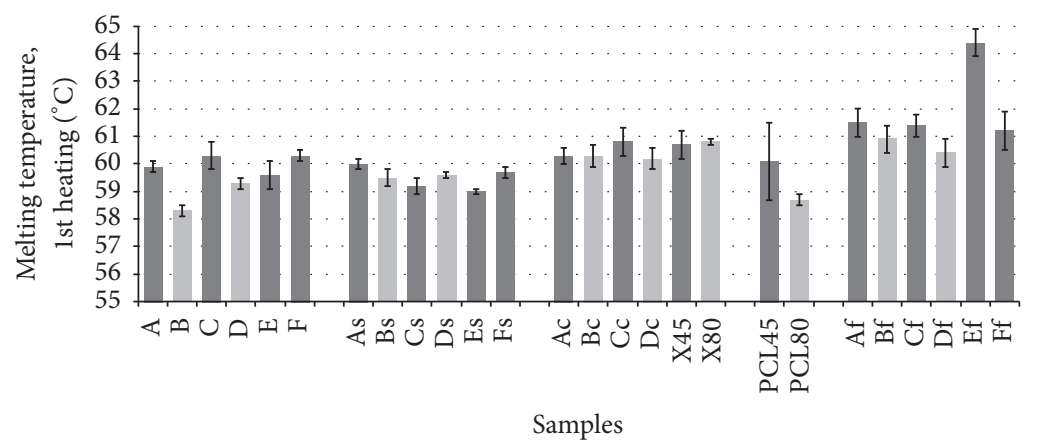

FIGURE 5: Graph illustrating melting temperature for all the materials tested by DSC analysis. The melting temperature was taken from the 1st heating curve. The light gray columns representing materials produced from PCL of $M_{n}=80,000 \mathrm{~g} / \mathrm{mol}$; the dark gray columns representing materials produced from PCL $M_{n}=45,000 \mathrm{~g} / \mathrm{mol}$. Error bars represent $95 \%$ confidence intervals.

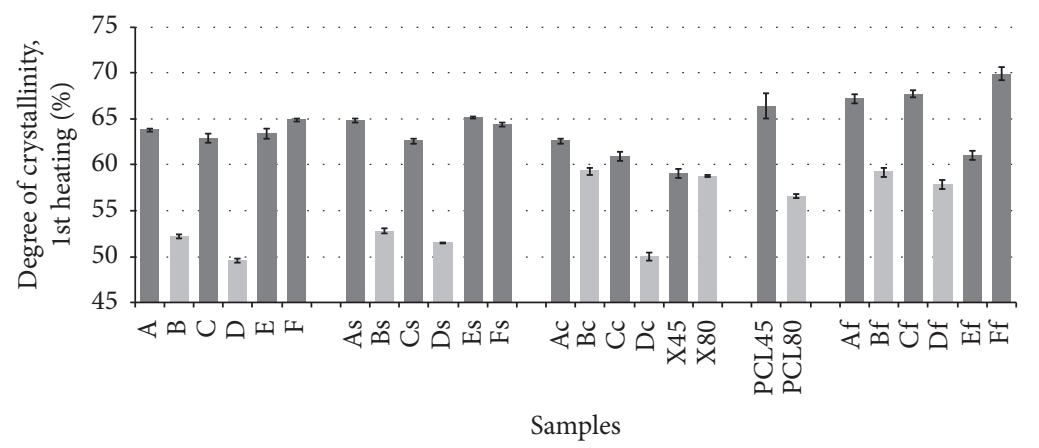

FIGURE 6: Graph illustrating degree of crystallinity for the all PCL materials tested by DSC analysis. The degree of crystallinity was calculated from the 1st heating curve. The light gray columns representing materials produced from PCL of $M_{n}=80,000 \mathrm{~g} / \mathrm{mol}$; the dark gray columns representing materials produced from PCL $M_{n}=45,000 \mathrm{~g} / \mathrm{mol}$. Error bars representing $95 \%$ confidence intervals.

\section{Acknowledgments}

The support for this research was provided by the Project Nanofiber Materials for Tissue Engineering, Reg. number CZ.1.05/3.1.00/14.0308, which is cofinanced by the European Social Fund and the State Q3 Budget of the Czech Republic and by the Ministry of Education, Youth and Sports in the framework of the targeted support of the National Programme for Sustainability I LO 1201 and Developlmet Project of TUL 2014 DZ TUL 2011-15, Chapter.2.1.

\section{References}

[1] E. Piorkowska and G. C. Rutledge, Handbook of Polymer Crystallization, John Wiley \& Sons, Inc., Hoboken, NJ, USA, 2013.

[2] M. Bognitzki, W. Czado, T. Frese et al., "Nanostructured fibers via electrospinning," Advanced Materials, vol. 13, no. 1, pp. 7072, 2001.

[3] D. H. Reneker and A. L. Yarin, "Electrospinning jets and polymer nanofibers," Polymer, vol. 49, no. 10, pp. 2387-2425, 2008.

[4] D. H. Reneker, A. L. Yarin, H. Fong, and S. Koombhongse, "Bending instability of electrically charged liquid jets of polymer solutions in electrospinning," Journal of Applied Physics, vol. 87, no. 9, pp. 4531-4547, 2000.
[5] A. A. Shah, F. Hasan, A. Hameed, and S. Ahmed, "Biological degradation of plastics: a comprehensive review," Biotechnology Advances, vol. 26, no. 3, pp. 246-265, 2008.

[6] M. A. Woodruff and D. W. Hutmacher, "The return of a forgotten polymer-polycaprolactone in the 21st century," Progress in Polymer Science, vol. 35, no. 10, pp. 1217-1256, 2010.

[7] T. K. Dash and V. B. Konkimalla, "Poly- - -caprolactone based formulations for drug delivery and tissue engineering: a review," Journal of Controlled Release, vol. 158, no. 1, pp. 15-33, 2012.

[8] O. Ero-Phillips, M. Jenkins, and A. Stamboulis, "Tailoring crystallinity of electrospun PLLA fibres by control of electrospinning parameters," Polymers, vol. 4, no. 3, pp. 1331-1348, 2012.

[9] D. Kołbuk, P. Sajkiewicz, and T. A. Kowalewski, "Optical birefringence and molecular orientation of electrospun polycaprolactone fibers by polarizing-interference microscopy," European Polymer Journal, vol. 48, no. 2, pp. 275-283, 2012.

[10] H.-W. Tong and M. Wang, "An investigation into the influence of electrospinning parameters on the diameter and alignment of poly(hydroxybutyrate-co-hydroxyvalerate) fibers," Journal of Applied Polymer Science, vol. 120, no. 3, pp. 1694-1706, 2011.

[11] D. Lukáš, A. Sarkar, L. Martinová et al., "Physical principles of electrospinning (electrospinning as a nano-scale technology of the twenty-first century)," Textile Progress, vol. 41, no. 2, pp. 59$140,2009$.

[12] T. J. Sill and H. A. Von Recum, "Electrospinning: applications in drug delivery and tissue engineering," Biomaterials, vol. 29, no. 13, pp. 1989-2006, 2008. 
[13] Y. Lu, Y. Li, S. Zhang et al., "Parameter study and characterization for polyacrylonitrile nanofibers fabricated via centrifugal spinning process," European Polymer Journal, vol. 49, no. 12, pp. 3834-3845, 2013.

[14] D. Lubasova and L. Martinova, "Porous polycaprolactone nanofibers prepared by electrospinning," in Proceedings of the 1st Conference with International Participation (NANOCON '09), Roznov pod Radhostem, Czech Republic, October 2009.

[15] E. Kostakova, M. Seps, P. Pokorny, and D. Lukas, "Study of polycaprolactone wet electrospinning process," Express Polymer Letters, vol. 8, no. 8, pp. 554-564, 2014.

[16] D. S. Rosa, C. G. F. Guedes, and M. A. G. Bardi, "Evaluation of thermal, mechanical and morphological properties of PCL/CA and PCL/CA/PE-g-GMA blends," Polymer Testing, vol. 26, no. 2, pp. 209-215, 2007.

[17] N. E. Zander, "Formation of melt and solution spun polycaprolactone fibers by centrifugal spinning," Journal of Applied Polymer Science, vol. 132, no. 2, Article ID 41269, 2015.

[18] R. N. McElhaney, "The use of differential scanning calorimetry and differential thermal analysis in studies of model and biological membranes," Chemistry and Physics of Lipids, vol. 30, no. 2-3, pp. 229-259, 1982.

[19] T. McNally and P. Pötschke, Polymer-Carbon Nanotube Composites: Preparation, Properties and Applications, Woodhead Publishing, 1st edition, 2011. 

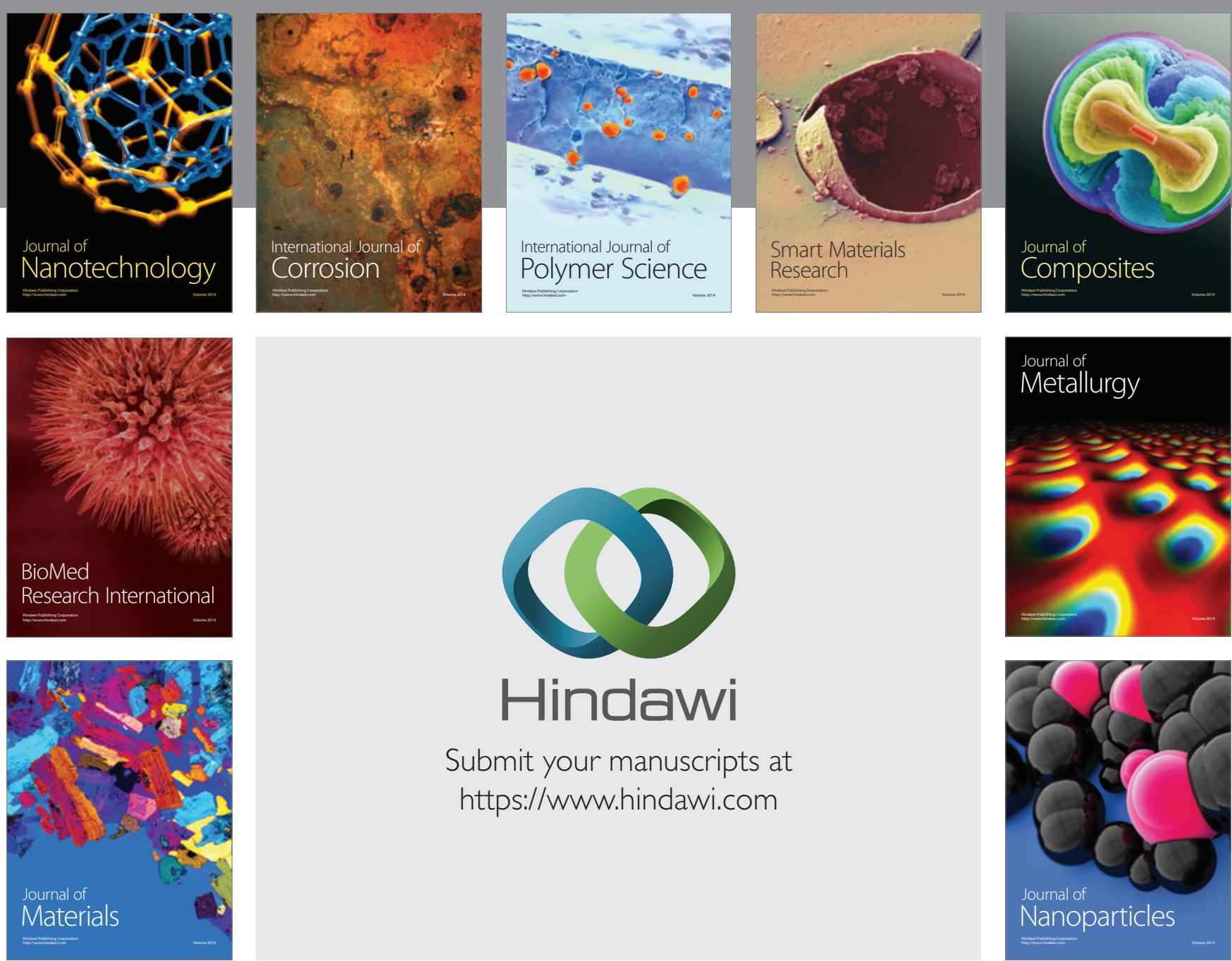

\section{Hindawi}

Submit your manuscripts at

https://www.hindawi.com

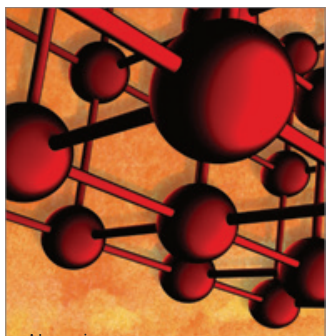

Materials Science and Engineering
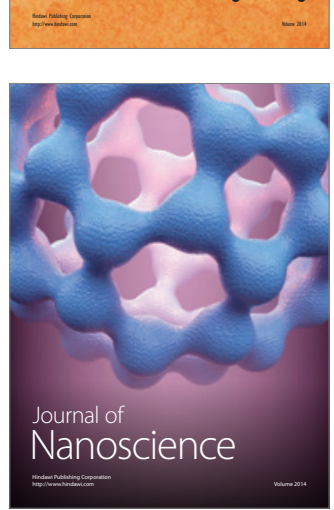
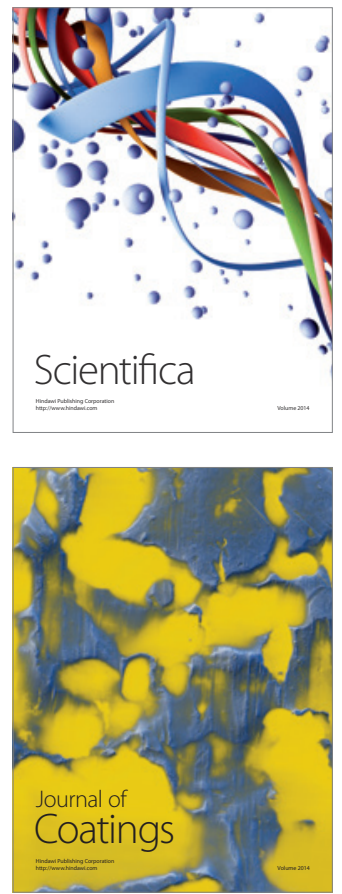
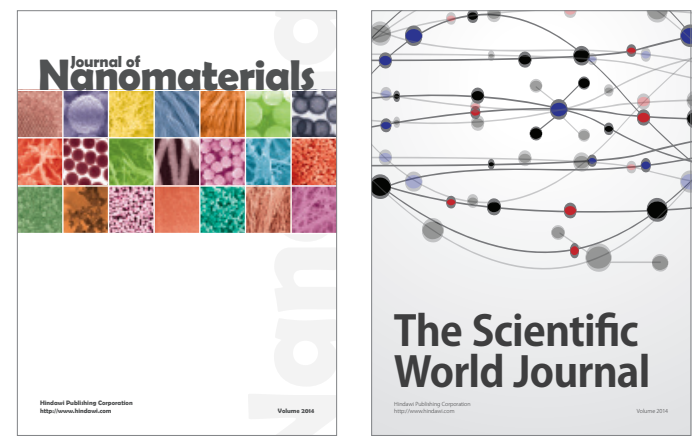

The Scientific World Journal
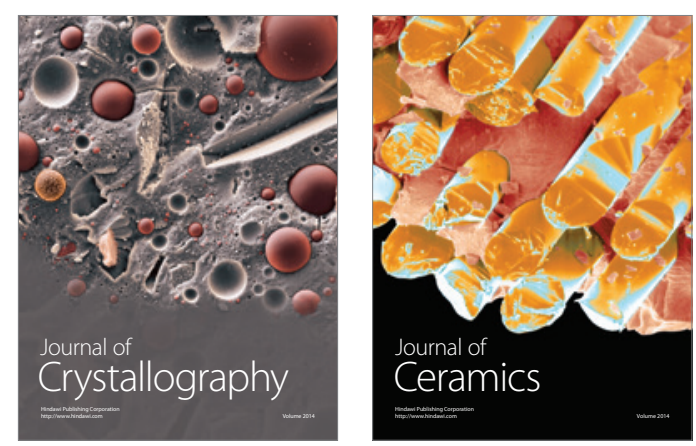
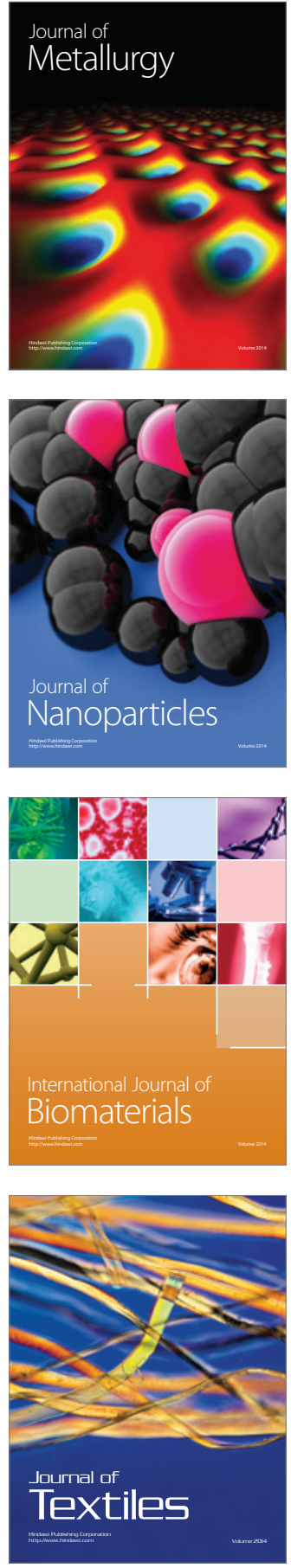